\title{
Near quenching limit instabilities of concurrent flame spread over thin solid fuel
}

\section{Shuangfeng Wang, Suide Wang, Kangcheng Zhu, Yuan Xiao \& Zhanbin Lu}

To cite this article: Shuangfeng Wang, Suide Wang, Kangcheng Zhu, Yuan Xiao \& Zhanbin Lu (2015): Near quenching limit instabilities of concurrent flame spread over thin solid fuel, Combustion Science and Technology, DOI: 10.1080/00102202.2015.1125346

To link to this article: http://dx.doi.org/10.1080/00102202.2015.1125346

Accepted author version posted online: 15 Dec 2015.

Submit your article to this journal

III Article views: 13

Q View related articles $\asymp$

View Crossmark data $\asymp$ 


\section{Near quenching limit instabilities of concurrent flame spread over thin solid fuel}

(Manuscript submitted to Combustion Science and Technology)

Shuangfeng Wang

Institute of Mechanics, Chinese Academy of Sciences, Beijing, China 100190 E-mail address:

sfwang@imech.ac.cn

Suide Wang

Institute of Mechanics, Chinese Academy of Sciences, Beijing, China 100190 E-mail address: wangsuide@sina.com

Kangcheng Zhu

Institute of Applied Mathematics and Mechanics, Shanghai University, Shanghai, China 200072

E-mail address: colinzkc@163.com

Yuan Xiao ${ }^{1}$

Institute of Mechanics, Chinese Academy of Sciences, Beijing, China 100190 E-mail address: yuan.xiao@my.jcu.edu.au

${ }^{1}$ Current address: College of Science, Technology and Engineering, James Cook University, Townsville, QLD 4811, Australia

Zhanbin Lu 
Institute of Applied Mathematics and Mechanics, Shanghai University, Shanghai, China 200072

E-mail address: luzhanbin@gmail.com

Near quenching limit instabilities of concurrent flame spread over thin solid fuel

December 10, 2015

\begin{abstract}
Near quenching limit instabilities of concurrent flame spread over thin cellulosic fuel are experimentally studied by employing a narrow channel apparatus. Depending on the oxygen concentration of the imposed flow, two different kinds of instabilities have been identified. Specifically, for oxygen concentration below a critical value, the instability is of fingering or cellular type, whereas for supercritical oxygen concentrations, travelling wave instability prevails, characterized by transverse creeping motion of the flamelets across the fuel edge. Both instabilities are usually accompanied by recurrent flamelet growing and splitting during the flame spread processes. It is justified that the two kinds of instabilities herein identified are diffusive-thermal in nature and may be classified into the category of near quenching limit instability of non-adiabatic diffusion flames. Further, an attempt has been made to gain insight into the physical mechanisms of the flame instabilities by exploiting the similarities between flame spread and smolder wave propagation.
\end{abstract}

Keywords: Concurrent flame spread; Thin solid fuel; Smolder wave; Near quenching limit; Fingering instability; Travelling wave instability 


\section{Introduction}

As a unique configuration of moving diffusion flame combustion, flame spread over solid fuel constitutes the prototype model of many real life fire spread processes, ranging from relatively small scale room fires to large scale wild forest fires, and therefore has been extensively studied in the past several decades; see, e.g., the review articles (de Ris 1968; Di Blasi 1993; FernandezPello 1984; Fernandez-Pello and Hirano 1983; T'ien et al. 2001; Wichman 1992; Williams 1977). Owing to the inherent complexity involved, the study of flame spread over solids has been classified into different sub-categories in the literature. Specifically, from the viewpoint of the gas phase, flame spread is said to be countercurrent or opposed flow if the flame spreads against the imposed oxidizer flow; otherwise, if the flame spreads in the same direction as the imposed flow, it is designated as concurrent or flow-assisted. On the other hand, from the perspective of the solid phase, there exist two limiting cases with regard to the thickness of the solid fuel, namely thermally thin if the fuel is heated uniformly throughout its thickness by the spreading flame, and thermally thick if solid heat conduction fails to penetrate the entire fuel thickness such that significant temperature gradients develop along the direction normal to the fuel surface. Consequently, most existing works on flame spread over solid fuel fall into four distinct regimes. The present work is concerned with the concurrent, thermally thin regime.

Besides flaming, there exists an alternative mode of combustion over solid fuel, namely smoldering (Ohlemiller 1985; Rein 2009), whose connection with flame spread has been first explored by Ohki and Tsugé (1974). Despite the fundamental difference in chemical reaction, these two modes of solid combustion actually have much in common so far as the structural 
characteristics and the involved physical mechanisms are concerned. Particularly, similar to flame spread, a smolder front can propagate either against or in the same direction as the imposed oxidizer flow, and likewise, the former is called an opposed flow or reverse smolder wave, whereas the latter is called a concurrent or forward smolder wave (Ohlemiller and Lucca 1983).

In reality, both flame spread and smolder propagation processes are inevitably subjected to external heat losses, the origin of which includes radiation, conduction, convection or a combination of these mechanisms. The impact of heat loss is in general not appreciable for relatively higher flow velocities. However, it becomes progressively more pronounced when the flow velocity is substantially decreased, until quenching occurs at a limiting velocity (Akkutlu and Yortsos 2003; Kumar et al. 2003; Lu and Dong 2011; Olson 1991; Olson et al. 1988). For the case of flame spread, there exists an opposite extinction limit, namely the high flow velocity blow-off limit, which is induced by the finite kinetics effect, or in other words, the finite Damköhler number effect. With the decrease of the oxygen concentration, the quenching limit and the blow-off limit eventually join together at a critical oxygen concentration, below which self-sustained flame spread cannot occur for any flow velocities, thereby defining an absolute inflammability limit.

In a microgravity experiment aboard space shuttle, Olson et al. (1998) found that, when smoldering combustion was initiated at the center of a thin cellulosic fuel, the part of the smolder front that propagated upstream eventually spawned a large population of fingerlike smolder fronts. Such an unusual phenomenon has not been observed in a repetition of the same 
experiment under $1 g$ environment, suggesting that the fingering propagation mode might be an exclusive phenomenon prevailing in the near quenching limit region, which was inaccessible under $1 g$ environment due to the presence of buoyant convection. At almost the same time, similar fingering propagation mode of reverse smolder combustion over thin cellulosic fuel was identified independently by Zik and Moses (1998, 1999) and Zik et al. (1998), by employing a narrow channel apparatus to simulate the microgravity conditions. Furthermore, the characteristic scales of the developed fingering state and their relationship with the control factors were extensively discussed.

Various models of smolder combustion have been developed to account for the fingering pattern found in the microgravity experiments. $\mathrm{Lu}$ and Yortsos (2005) used a 2-D porenetwork model to simulate the porous media and captured the formation of fingering pattern in reverse filtration combustion. By employing a simplified diffusive-thermal model, Lu et al. (2006) and Yuan and Lu (2013) numerically studied the stability of reverse smolder waves, and identified fingering (or cellular) and travelling wave instabilities on the fuel-rich and fuel-lean solution branches, respectively. Ijioma et al. $(2013,2015)$ developed two macroscopic models for reverse smolder combustion through a formal homogenisation approach, and by choosing appropriate Peclet and Lewis numbers, successfully reproduced various fingering patterns observed in the experiments of Zik and Moses (1999). Uchida et al. (2015) examined the effects of Lewis number and convection on reverse smolder combustion by using a quasi-two-dimensional model, and concluded that the Lewis number must be considerably smaller than unity for the fingering pattern to develop; further, it was confirmed that convection is not an essential factor to produce a fingering pattern. All these findings substantiate the view that, similar to the case of cellular 
premixed flames, the fingering smolder fronts observed in the microgravity experiments were due to diffusive-thermal instability of the reverse smolder waves, in which the Lewis number plays an essential role.

Inspired by the findings from the microgravity smolder experiments, Olson et al. (2009) conducted experiments on opposed flow flame spread over thin solid fuel under actual (in drop tower) and simulated (in narrow channel apparatus) microgravity conditions. Similar to the smolder case, it was observed that the initially continuous flame front broke into separate flamelets when the imposed oxidizer flow velocity was reduced near to the quenching limit, leaving behind a fingering pattern on the fuel surface. The statistical characteristics of the flamelet population had been studied by Olson et al. (2006) via the logistic model. In a downward flame spread experiment under normal gravity condition, Zhang et al. (1992) showed that reducing the oxygen Lewis number eventually led to the onset of cellular instability, suggesting that diffusive-thermal instability is still the mechanism that is responsible for the fingering patterns observed in the flame spread mode. Kagan and Sivashinsky (2008) developed a 2-D free-interface model for opposed flow flame spread over thin solid fuel, from which a nonlinear equation was derived to describe the evolution of the disturbed flame front. Numerical simulations of the nonlinear evolution equation produced fingering patterns that agree favorably with those obtained from the experiments of Zik and Moses (1999).

The cellular and fingering instabilities identified so far in the experiments, either in smolder or flame spread mode, have been confined to the opposed flow regime. As for the concurrent regime, to the best of the authors' knowledge, there has been no systematic experimental study 
that is exclusively devoted to the stability of forward smolder wave or concurrent flame spread. Nevertheless, it is worth mentioning that several fragments exist in the literature. In the microgravity experiment of Olson et al. (1998) on smolder front propagation, it was found that sporadically some downstream smolder fronts survived and propagated downwind, albeit much more slowly than the upwind propagating ones. This may be regarded as an evidence of fingering instability in forward smolder combustion. In Zik and Moses (1999), a short subsection has been devoted to the concurrent (coflow) regime, for which the experimental results showed that the smolder front was more stable in comparison with the opposed flow regime, and although transient fingers might appear, they eventually merged and a continuous front resulted. Olson et al. (2006) described an accidental experimental finding: in an opposed flow flame spread experiment conducted in narrow channel, after the flamelets reached the upstream edge of the fuel sample, instead of going out, the flame fronts turned around and resumed burning in the form of concurrent flame spread. Surprisingly, the concurrent flamelets underwent transverse creeping motion, traversing the upstream edge of the unburned fuel sample. Since that paper is predominantly concerned with the statistical characteristics of the flamelets, especially the fingering flamelets formed in opposed flow regime, the issue as to why and under what conditions can the concurrent flamelets develop has not been addressed.

In the reference frame that moves with the propagating (spreading) reaction front, both forward smolder wave and concurrent flame spread are structurally analogous to the canonical, stationary, diffusion flame, and hence it is expected that their stability will share many common characteristics with that of diffusion flames. Chen et al. (1992) experimentally studied the diffusive-thermal instability of diffusion flames, and identified cellular instability near the blow- 
off limit. Subsequently, cellular and pulsating instabilities of diffusion flames near the blow-off limit have been extensively studied (Cheatham and Matalon 1996, 2000; Kim 1997; Kim and Lee 1999; Kim et al. 1996; Kukuck and Matalon 2001; Metzener and Matalon 2006; Sohn et al. 1999). In addition to the low Damköhler number blow-off limit, when heat loss effect is incorporated, the diffusion flame may develop a quenching limit at large Damköhler numbers. Within the context of a diffusive-thermal formulation, Sohn et al. (2000) and Miclavcic et al. (2005) examined the stability characteristics near the quenching limit for a model diffusion flame problem, and identified bifurcations that may lead to limit cycle solutions, thereby corresponding to onset of sustained flame oscillations. A detailed account of the instabilities of diffusion flames is available in the review by Matalon (2009).

Compared with the case of gaseous fuel diffusion flames, theoretical studies that address the instabilities of concurrent flame spread or forward smolder waves are relatively rare. Aldushin and Matkowsky (1998) and Brailovsky et al. (2008) analytically studied the hydrodynamic instability of forward smolder combustion, predicting that fingering instability may develop, resulting from porosity increase in the solid phase. In a numerical study on diffusive-thermal instability of non-adiabatic forward smolder waves, Lu and Dong (2011) predicted that fingering instability may develop when the imposed oxidizer flow velocity is sufficiently close to the quenching limit, where heat loss plays a dominant role.

The numerical results of Lu and Dong (2011) directly motivate the present study, which is partly aimed at providing an experimental comparison with their numerical predictions in the context of concurrent flame spread. Besides that, the ultimate objective of the present work is to obtain an 
overall picture of the dynamical behavior of concurrent flame spread near the quenching limit, by conducting systematic experiments that cover a comprehensive range of parameters.

\section{Experiment}

In order to suppress buoyant convection, following Zik et al. (1998) and Olson et al. (2009), we developed a narrow channel apparatus for experimental study of the stability characteristics of concurrent flame spread. As shown schematically in Fig. 1, the narrow channel apparatus consists of two horizontally placed, parallel, quartz glass plates with a fixed spacing of $11 \mathrm{~mm}$. The lateral sides of the channel are sealed, while the downstream end is open, and the upstream end is connected to the outlet of a gas supply unit, where the supplied oxygen (or nitrogen) and air are mixed at appropriate flow rates controlled by mass flow controllers. The metered gas mixture is led to flow successively through a honeycomb gas diffuser and a contraction section, to ensure uniformity of the oxidizer flow before entering the narrow channel.

For all the tests reported in this paper, Whatman No. 41 quantitative filter paper was used as the solid fuel sample, which had a typical thickness of $215 \mu \mathrm{m}$ and an area density of $84 \mathrm{~g} / \mathrm{m}^{2}$. In some tests (not reported in this paper) the fuel has been replaced by Whatman No. 40 (thickness: $210 \mu \mathrm{m}$, area density: $92 \mathrm{~g} / \mathrm{m}^{2}$ ) or No. 1 (thickness: $180 \mu \mathrm{m}$, area density: $88 \mathrm{~g} / \mathrm{m}^{2}$ ) series, but no significant difference was found in the experimental phenomena, so it would seem reasonable to believe that the experimental results presented herein are not specific to the cellulosic fuel used. Before each test, the fuel sample was first taped to the side frames of a stainless steel sample holder, and was kept slightly stretched by applying a spring at the downstream end of the sample holder, to prevent the fuel sample from thermal-induced deformation during the burning process. 
The fixed fuel sample had an effective width of $150 \mathrm{~mm}$ and a length of $254 \mathrm{~mm}$. A resistively heated ignition wire was placed at the upstream end of the sample holder, and was made in uniform contact with the sample edge to ensure uniform ignition across the sample. Then, the sample holder set was carefully pushed into the narrow channel and was placed symmetrical with respect to the center line of the channel, in order to avoid the inhomogeneities produced by the channel side walls. Prior to this transfer procedure, the sample holder frames were adjusted outside the channel so that the fuel sample would be kept right on the middle plane between the top and bottom plates in the channel. During each test, ignition was initiated after the gas flow was fully developed. Then, the igniter was switched off once stable flame propagation was observed and the flame was completely detached from the ignition wire. The flame spread process was recorded by a color digital video camera placed above the top plate.

Oxygen concentration and flow velocity of the imposed oxidizer flow are conjectured to be the key parameters that influence the stability characteristics of concurrent flame spread, so both of them have been varied over a sufficiently wide range in the experiment. The gas flow velocity is represented by the average flow velocity across the cross-section of the channel, which is calculated based on the preset gas flow rates and the cross-sectional area of the channel. In all the tests herein reported, air was chosen as a gas source to mix with oxygen or nitrogen according to the specified flow rate proportion to achieve higher or lower oxygen concentration. All tests were conducted under the local ambient pressure condition. 


\section{Experimental results}

\subsection{Fingering or cellular regime}

Fig. 2 shows the evolution process of flame spread in an $\mathrm{N}_{2} /$ Air atmosphere with oxygen concentration $C_{\mathrm{Of}}=15 \%$ (here and hereinafter, volume percent) and gas flow velocity $U=6$ $\mathrm{cm} / \mathrm{s}$, represented by sequential snapshots taken from the video record. As can be seen, shortly after ignition, the initially continuous flame front breaks into several discrete flamelets, which spread downwind together and leave behind a fingering burned-out pattern. It is evident that each flamelet assumes an arc shape, with the convex surface facing downstream. Moreover, the arcshaped flamelet grows in width as it moves forward, and once its width exceeds a critical value,

the flamelet splits in the middle into two children flamelets, resulting in a bifurcation of the trailing finger tip. Unfortunately, as can be seen from Fig. 2, all the second-generation children flamelets go out eventually. A test with an even lower velocity, $U=5 \mathrm{~cm} / \mathrm{s}$, has been carried out subsequently, but it turned out that the initiated flame front failed to spread forward and extinguished soon after ignition. This suggests that the quenching limit for the current oxygen concentration should lie between 5 and $6 \mathrm{~cm} / \mathrm{s}$.

Fig. 3 shows the flame evolution process for $U=7 \mathrm{~cm} / \mathrm{s}$ and $C_{\mathrm{Of}}=15 \%$. It is seen that, after a short transient, the initially continuous flame front breaks into three isolated flamelets, of which only one is survived. Subsequently, this single flamelet undergoes a series of very regular, period-doubling-like bifurcation process, which is characterized by recurrent flamelet growing 
and splitting, along with random merging and extinction of the children flamelets. Eventually, the survived children flamelets succeed in burning through the fuel sample, leaving behind a tree-like burned-out pattern. Note that such a period-doublinglike bifurcation mode arising in concurrent flame spread is qualitatively reminiscent of the front splitting mode identified in the experiments of reverse smolder (Olson et al. 1998; Zik and Moses 1998, 1999; Zik et al. 1998) and opposed flow flame spread (Olson et al. 2006, 2009), although the gas flow directions are opposite in the two situations. This suggests that the currently identified fingering or cellular regime of concurrent flame spread may also be a manifestation of the diffusive-thermal mechanism; or more specifically, it could be the post-development of the flame front beyond the onset of diffusive-thermal instability, as an adaptation to the oxygen-deficient environment. Further discussions on this issue will be postponed to the next Section.

The period-doubling-like bifurcation character of concurrent flame spread can be more clearly seen through the bifurcation diagram shown in Fig. 4, which is delineated for the same case shown in Fig. 3, by tracking the positions of the flamelets during their evolution processes. The flamelet position is identified with the midpoint along the flamelet arc, and the error in resolving it from the flame images is estimated to be within 3 pixels $(\approx 1 \mathrm{~mm})$ in both directions. As can be seen, the bifurcation diagram displays an apparent tree pattern-the flamelets grow and split repeatedly so as to maximize their intake of oxygen from the gas stream, just like tree branches extending and bifurcating to make the leaves gain more exposure to sunlight. It is evident that the more inclined the flamelet spread trajectory is to the gas flow direction, the more chance it gets to survive. 
Fig. 5 plots the time evolution of the longitudinal flame position and the flamelet width for branch $O C$ as labeled in Fig. 4. It is seen that, although influenced by the interactions with the neighboring branches, the position vs. time data along branch $O C$ exhibit a quasi-linear feature, and a linear fitting gives a flame spread rate $V_{\mathrm{f}} \approx 0.18 \mathrm{~cm} / \mathrm{s}$. The evolution of the flamelet width, which is defined as the linear distance between the two edges of the flamelet arc, exhibits a recurrent growing-dropping pattern: in each cycle, the width grows until it reaches a peak value, a critical width at which the flamelet splits, manifesting as a sudden drop of the flamelet width. Due to the complex interactions with the neighboring branches, the critical flamelet width and the duration of each cycle are not constant.

Fig. 6 shows the case for gas flow velocity $U=9 \mathrm{~cm} / \mathrm{s}$, still at $15 \%$ oxygen. In comparison with the previous two cases, although the flamelets still exhibit cellularity and flamelet growing and splitting are still present, there is an appreciable decrease in the average spacing between neighboring flamelets, and as a result their interactions prevent the formation of fingered pattern behind the flamelets.

In Fig. 7, the gas flow velocity is further increased to $17 \mathrm{~cm} / \mathrm{s}$, with the oxygen concentration still remaining unchanged. It is evident that, under such a high flow velocity, the visible flame length is substantially increased in comparison with previous low flow velocity cases. Moreover, the flame front becomes fragmented soon after ignition, but compared with previous low velocity cases, the evolution of the fragmented fronts appears very irregular. Particularly, the fragmented fronts do not exhibit regular cell growth and split, which has been found to be characteristic of flamelet evolution in previous low flow velocity cases. This is an indication that the diffusive- 
thermal mechanism that used to be dominant in the low velocity cases are now taken over by the hydrodynamic effects. In this connection, it seems reasonable to attribute the fragmentation of the flame front to local extinction of the diffusion flame, which is induced by hydrodynamic disturbances, rather than by inherent diffusive-thermal instability.

Fig. 8 plots the flame spread rate and critical flamelet width as a function of gas flow velocity for oxygen concentration $C_{\mathrm{Of}}=15 \%$. The flame spread rate data are obtained in a similar way as the case of $U=7 \mathrm{~cm} / \mathrm{s}$, as is demonstrated in Fig. 5, by linearly fitting the positions of the fastest spreading flamelets as a function of time. It is evident that the flame spread rate increases monotonically with the gas flow velocity for the range considered. This is expected, since increasing the gas flow velocity on one hand enhances the supply of oxygen to the flame front, and on the other hand reduces the relative heat loss intensity, both tending to increase the flame temperature, and hence the flame spread rate. There is a scatter in the data of critical flamelet width corresponding to each gas flow velocity, due to the complex interactions involved during the evolution of the flamelets. The statistical average and standard deviation for the data of critical flamelet width are obtained at each gas flow velocity except the two cases $U=15$ and 17 $\mathrm{cm} / \mathrm{s}$, for which flamelet growth and split are virtually no longer discernible. As can be seen, except very near to the quenching limit, the average critical flamelet width shows a tendency to increase with the gas flow velocity.

\subsection{Transverse creeping regime}

Fig. 9 shows a sequence of snapshots taken from the test for concurrent flame spread in air (oxygen concentration $C_{\mathrm{Of}}=21 \%$ ), with imposed flow velocity $U=3 \mathrm{~cm} / \mathrm{s}$. As can be seen, after 
an initial developing stage, the continuous flame front is survived by a single flamelet. Then, in the subsequent development, similar to the previous fingering or cellular regime, this single flamelet experiences recurrent growing and splitting, spawning a train of children flamelets that distribute along the unburned fuel edge. However, different from the fingering or cellular regime, each of the flamelets undergoes an essentially transverse creeping motion, traversing the fuel edge often in a back and forth manner, quite like a creeping caterpillar that nibbles the edge of a leaf. As a consequence, the unburned fuel maintains a relatively smooth edge at all times and there is no leftover trailing behind the flamelets. It is worth mentioning that this transverse creeping regime is essentially consistent with the experimental observations of concurrent flame spread described by Olson et al. (2006).

The above flame evolution process can be best visualized through Fig. 10, where transverse edge positions of the flamelets are tracked with time. Similar to Fig. 4, Fig. 10 also exhibits a tree pattern. However, since Fig. 10 has time as the vertical coordinate, it visualizes how the flamelets grow, split and meander along the transverse direction with time. To get the information regarding flame spread in the longitudinal direction, the time history of longitudinal position of the unburned fuel edge is tracked along a fixed vertical line, which is located at $y=$ $5.49 \mathrm{~cm}$ in Fig. 10, and the result is illustrated in Fig. 11. Fig. 10 shows that the passage of flamelets through this fixed $y$ coordinate is intermittent. Correspondingly, the relative position versus time plot in Fig. 11 forms a step-like pattern. Accordingly, there are two flame spread rates that may be defined: the first counts the total elapsed time, by linearly fitting the entire position vs. time data, and hence may be called the nominal flame spread rate; the second counts only the time portions when the flamelets are present, by linearly fitting only the slope portions 
(the three solid line segments in Fig. 11) of the step-like fuel edge position data, and is referred to as the actual flame spread rate. The fitting results show that, for the current case, the nominal flame spread rate $V_{\mathrm{fn}} \approx 0.019 \mathrm{~cm} / \mathrm{s}$, whereas the actual flame spread rate $V_{\mathrm{fa}} \approx 0.047 \mathrm{~cm} / \mathrm{s}$. As stated before, the unburned fuel maintains a relatively smooth edge during the flame evolution, so the measured nominal and actual flame spread rates are considered to be representative of the global ones.

In Fig. 12, sequential images are shown for concurrent flame spread in air, with the gas flow velocity increased to $5 \mathrm{~cm} / \mathrm{s}$. It is seen that, similar to the previous case for $U=3 \mathrm{~cm} / \mathrm{s}$, initially the continuous flame front breaks into a train of discrete flamelets, which subsequently undergo a transverse creeping motion along the fuel edge. However, as time goes on, the flamelets gradually join together such that the continuous flame front is recovered. When the flow velocity was increased further in the experiment, the flame front remained continuous and no breakup or transverse motion was observed, so $U=5 \mathrm{~cm} / \mathrm{s}$ may be considered to mark the transition from the continuous flame front regime to the transverse creeping regime for concurrent flame spread in air. Along the same line of reasoning as in the fingering or cellular regime, transverse creeping motion of flamelets across the fuel edge might be a manifestation of the post-development of flame front beyond the onset of travelling wave instability.

\subsection{Flammability map and stability diagram}

Following the typical cases presented above, a series of concurrent flame spread tests have been conducted in the narrow channel, with the oxygen concentration covering the range from $13 \%$ to 23\%. The experimental results are summarized in the flammability map as shown in Fig. 13. 
Consistent with the computational results of Kumar et al. (2003), the flammability boundary obtained in the present experiment for concurrent flame spread over thin solid fuel exhibits a Ushaped curve, with the interior corresponding to the flammable region. The left branch of the flammability boundary corresponds to the quenching limit, near which heat loss plays a significant role; the right branch is believed to correspond to the blow-off limit, which, due to the high flow velocities involved therein, and considering the focus of the present study (flame behavior near the quenching limit), has not been delineated except for very low oxygen concentrations. With the decrease in oxygen concentration, the two branches join together at a critical point, thereby defining an absolute inflammability limit, below which concurrent flame spread cannot be sustained for any flow velocities. Under the current experimental conditions, the oxygen concentration that corresponds to the absolute inflammability limit is determined to be between $13 \%$ and $14 \%$.

A thorough examination of the experimental record indicates that the entire flammable region may be divided into three sub-regions by two lines, with each of the sub-regions corresponding to a distinct flame spread regime. Specifically, as shown in Fig. 13, the first dividing line corresponds to the marginal stability boundary, which separates a region that is characterized by essentially continuous flame fronts spreading at uniform speeds, from regions where the flames suffer different kinds of instabilities. The marginal stability boundary lies very close to the quenching limit for higher oxygen concentrations and progressively gets farther from the left flammability boundary as the oxygen concentration goes down towards the absolute inflammability limit, implying that the flame spread process is more susceptible to flame instabilities in low oxygen environments. It should be pointed out that, in the experiment the 
transition between stable and unstable flame spread regimes was in general not abrupt, thereby rendering unambiguous identification of the transitional gas flow velocity very difficult. The situation becomes even worse for low oxygen concentration cases because then the transition tends to occur at high flow velocities, in which cases, as previously discussed, the hydrodynamic effects start to come into play and flame evolution may appear very irregular or even chaotic. To overcome this difficulty, the transition from unstable to stable flame spread is identified with the state where regular cell formation, growth and split are no longer discernible.

The second dividing line is a short line segment that corresponds to a critical oxygen concentration, $C_{\mathrm{Oc}}$, which is approximately $19 \%$ for the current configuration. This line divides the whole unstable region into two parts: below the critical oxygen concentration, the flame instability is of fingering or cellular type, whereas above the critical oxygen concentration, the flame instability is of travelling wave type, characterized by transverse creeping motion of the flamelets along the fuel edge. Both instabilities are usually accompanied by regular flamelet growth and split. To illustrate the transitional phase between these two instabilities, Fig. 14 presents a sequence of snapshots from the test with $C_{\mathrm{Of}}=19 \%$ and $U=5 \mathrm{~cm} / \mathrm{s}$. As can be seen, during the initial stage of the spread process, the flame evolution seems to follow the same trend as the previous low oxygen cases by disintegrating into a train of isolated flamelets, which then spread forward in the downwind direction. However, once a trailing fingered pattern is about to develop, the flamelets crawl back at once along the finger walls and swallow up the protrusions quickly, as is evidenced by the circled parts of Fig. 14. This burning mode repeats in the subsequent spread process, thus prohibiting the formation of fingered pattern on the fuel surface. 


\section{Discussions}

The physical mechanism underlying the identified near-limit flame behaviors may be understood within the framework of diffusive-thermal instability of diffusion flames. It is well-known that adiabatic diffusion flames are described by the S-shaped response curve (Liñán 1974), whose upper ignition branch approaches the Burke-Schumann limit at large Damköhler numbers and terminates at the blow-off limit at the other extreme of the Damköhler number. The BurkeSchumann limit turns out to be unconditionally stable (Cheatham and Matalon 2000; Kim et al. 1996), whereas near the blow-off limit the flame may suffer different kinds of instabilities, as has been discussed in the Introduction.

The S-shaped response of diffusion flame is altered once heat loss effect is incorporated (Sohn et al. 2000). Regardless of the origin of the heat loss, either radiative as in actual microgravity environment or conductive as in narrow channel, the overall heat loss intensity is directly correlated with the characteristic flame size, which is in turn inversely proportional to the imposed gas flow velocity. As a result, with the decrease in the flow velocity, the heat loss effect becomes progressively more pronounced, until quenching occurs at a limiting flow velocity. Consequently, in the presence of heat loss, the diffusion flame may have double extinction limits, corresponding to opposite extremes of the Damköhler number. The stability diagram in Fig. 13 indicates that the fingering (or cellular) and travelling wave instabilities identified in the current experiment are exclusive to the near quenching limit. Since the hydrodynamic effect is insignificant near the quenching limit and buoyancy has been inhibited by the narrow channel, it is justifiable that these two kinds of instabilities are diffusive-thermal in nature and may be 
classified into the broader category of diffusive-thermal instability of diffusion flames near the quenching limit, as a complement to the previously identified pulsating instability (Miclavcic et al. 2005; Sohn et al. 2000). The manifestation of different kinds of instabilities may be attributed to different bifurcations of the relevant parameters, e.g., the oxygen concentration, the fuel and oxidizer Lewis numbers, and so on.

As stated in the Introduction, despite their difference in chemical reaction routes, flame spread and smolder propagation over solid fuels share many common structural and dynamical characteristics. Therefore, further insight may be gained into the near-limit instabilities of concurrent flame spread by exploiting its similarities with forward smolder combustion. Lu and Dong (2011) numerically investigated the diffusive-thermal instability of non-adiabatic forward smolder waves, by taking convective heat loss into consideration. In their study, the oxygen mass fraction of the imposed oxidizer flow, $Y_{\mathrm{Of}}=23.5 \%$ (corresponding to a volume concentration $C_{\mathrm{Of}}$ $\approx 21 \%$ ), was chosen to be less than the stoichiometric coefficient of oxygen, $\mu=0.41$, so all the smolder waves considered therein were characterized by a reaction trailing structure. ${ }^{1}$ Their results indicate that fingering instability develops when the oxidizer flow velocity falls into a narrow range near the quenching limit. This is consistent with the current experimental finding

$<$ SUP $>1</$ SUP $>$ Strictly speaking, the terms 'reaction trailing' and 'reaction leading' are meaningful only in the context of adiabatic forward smolder combustion, wherein a persistent thermal wave structure is developed in front of or behind the reaction front (Schult et al. 1996). Nevertheless, we will retain these terms in the description of non-adiabatic forward smolder combustion, in the sense that the smolder wave would recover the corresponding adiabatic structure if heat loss were purged. 
of concurrent flame spread that, for oxygen concentration less than a critical value, $C_{\mathrm{Oc}} \approx 19 \%$, fingering or cellular instability prevails when the flow velocity approaches the quenching limit.

It should be noted that, despite the consistency in the conditions for the onset of fingering instability, the current experiment and the numerical simulations of Lu and Dong (2011) exhibit distinct features regarding the subsequent development of the reaction fronts. Specifically, for the flame spread experiment, as evidenced by the flame images shown in Figs. 2-3 and 6, beyond the onset of fingering instability, the developed flamelets have their convex surfaces facing downstream, rather than upstream as revealed by the simulation results of forward smolder combustion (Lu and Dong 2011). The second disparity pertains to the recurrent growing and splitting character of the fingered flamelets, which has been found to be characteristic of not only concurrent but also opposed flow flame spread for certain ranges of the parameters, but unfortunately has not been captured by the direct numerical simulation results of Lu and Dong (2011). It is postulated that such disparities may be attributed to the different flow configurations employed in the two situations, and in this connection employing a model that is similar to those used by Ijioma et al. (2013), Kagan and Sivashinsky (2008), and Uchida et al. (2015) might be a sensible choice for comparison purposes. Further studies are therefore needed to elucidate this issue.

For concurrent flame spread, Fig. 13 indicates that a bifurcation to travelling wave instability occurs once the oxygen concentration crosses the critical value $C_{\mathrm{Oc}}$. Correspondingly, for the simplified smolder model employed by Lu and Dong (2011), reaction leading structure takes over when the oxygen concentration is increased beyond the stoichiometric coefficient of 
oxygen. Akkutlu and Yortsos (2003) analyzed the structural characteristics of non-adiabatic forward smolder waves (aka in-situ combustion fronts) that have a reaction leading structure, by considering two different types of heat loss mechanisms, namely convective and conductive, respectively. Similar to the case of reaction trailing structure, a heat loss induced low flow velocity quenching limit was identified. Unfortunately, stability of the combustion fronts near this quenching limit has not been addressed in their study. To facilitate further comparison between the stability characteristics of flame spread and smolder propagation, research efforts along this line are therefore desirable.

\section{Conclusions}

Concurrent flame spread over thermally thin cellulosic fuel has been experimentally studied by employing a narrow channel apparatus, with emphasis placed on flame instabilities near the low flow velocity quenching limit. Results show that, when the imposed oxidizer flow velocity is above an oxygen concentration dependent threshold, the flame spread is usually characterized by a continuous flame front and a uniform spread rate, and is therefore deemed to be stable. By contrast, once the oxidizer flow velocity is reduced below the threshold, the flame front breaks into separate flamelets, thus marking the onset of flame instability. Two distinct types of instabilities are identified, namely fingering or cellular instability, which is characterized by cellular flame fronts with fingered burned-out pattern trailing behind, and travelling wave instability, which is characterized by transverse creeping motion of the flamelets along the unburned fuel edge. Both types of instabilities are usually accompanied by recurrent flamelet growing and splitting during the flame spread processes. A critical oxygen concentration, which 
is approximately $19 \%$ for the currently adopted fuel, is identified as a separation between these two instability regimes. Specifically, for oxygen concentrations below the critical value, the instability is of fingering or cellular type, whereas for supercritical oxygen concentrations travelling wave instability prevails.

It is justified that the instabilities identified in the current work can be classified into the broader category of diffusive-thermal instability of non-adiabatic diffusion flames near the quenching limit, as a complement to the previously identified pulsating instability. In addition, an alternative understanding of the flame instabilities has been pursued by exploiting the similarities between concurrent flame spread and forward smolder combustion, and in this connection it is found that the conditions for the onset of fingering instability in concurrent flame spread are in accord with the stability analysis results of forward smolder waves, which predict that fingering instability develops near the quenching limit for non-adiabatic forward smolder waves that have a reaction trailing structure.

\section{Acknowledgements}

This work is supported by the Strategic Pioneer Program on Space Science, Chinese Academy of Sciences, grant No. XDA04020410, the National Science Foundation of China, grant No. 50706024 and 11472167, and the Shanghai Leading Discipline Project. The support from the Key Laboratory of Microgravity, Institute of Mechanics, Chinese Academy of Sciences, is also gratefully acknowledged. We thank Ms. Feng Zhu for her help with some of the experiments. 


\section{References}

Akkutlu, I. Y. and Y. C. Yortsos (2003). "The dynamics of in-situ combustion fronts in porous media”. In: Combust. Flame 134, pp. 229-247.

Aldushin, A. P. and B. J. Matkowsky (1998). "Instabilities, fingering and the Saffman-Taylor problem in filtration combustion". In: Combust. Sci. Technol. 133, pp. 293-341.

Brailovsky, I., P. Gordon, and G. Sivashinsky (2008). "On flame-flow interaction in forward smoldering”. In: Combust. Flame 153, pp. 490-495.

Cheatham, S. and M. Matalon (1996). "Heat loss and Lewis number effects on the onset of oscillations in diffusion flames". In: Proc. Combust. Inst. 26, pp. 1063-1070.

- (2000). "A general asymptotic theory of diffusion flames with application to cellular instability". In: J. Fluid Mech. 414, pp. 105-144.

Chen, R., G. B. Mitchell, and P. D. Ronney (1992). "Diffusive-thermal instability and flame extinction in nonpremixed combustion”. In: Proc. Combust. Inst. 24, pp. 213-221.

de Ris, J. N. (1968). "The spread of a diffusion flame over a combustible surface". PhD Dissertation. Harvard University.

Di Blasi, C. (1993). "Modeling and simulation of combustion processes of charring and noncharring solid fuels". In: Prog. Energy Combust. Sci. 19, pp. 71-104. 
Fernandez-Pello, A. C. (1984). "Flame spread modeling”. In: Combust. Sci. Technol. 39, pp. 119-134.

Fernandez-Pello, A. C. and T. Hirano (1983). "Controlling mechanisms of flame spread". In: Combust. Sci. Technol. 32, pp. 1-31.

Ijioma, E. R., A. Muntean, and T. Ogawa (2013). "Pattern formation in reverse smouldering combustion: a homogenisation approach”. In: Combust. Theory Model. 17, pp. 185-223.

- (2015). "Effect of material anisotropy on the fingering instability in reverse smoldering combustion”. In: Int. J. Heat Mass Transfer 81, pp. 924-938.

Kagan, L. and G. Sivashinsky (2008). "Pattern formation in flame spread over thin solid fuels". In: Combust. Theory Model. 12, pp. 269-281.

Kim, J. S. (1997). "Linear analysis of diffusional-thermal instability in diffusion flames with Lewis numbers close to unity", In: Combust. Theory Model. 1, pp. 13-40.

Kim, J. S. and S. R. Lee (1999). "Diffusive-thermal instability in strained diffusion flames with unequal Lewis numbers”. In: Combust. Theory Model. 3, pp. 123-146.

Kim, J. S., F. A. Williams, and P. D. Ronney (1996). "Diffusional-thermal instability of diffusion flames". In: J. Fluid Mech. 327, pp. 273-301.

Kukuck, S. and M. Matalon (2001). "The onset of oscillations in diffusion flames”. In: Combust. Theory Model. 5, pp. 217-240. 
Kumar, A., H. Y. Shih, and J. S. T’ien (2003). “A comparison of extinction limits and spreading rates in opposed and concurrent spreading flames over thin solids”. In: Combust. Flame 132, pp. 667-677.

Liñán, A. (1974). "The asymptotic structure of counterflow diffusion flames for large activation energies". In: Acta Astronautica 1, pp. 1007-1039.

Lu, C. and Y. C. Yortsos (2005). "Pattern formation in reverse filtration combustion". In: Phys. Rev. E 72.036201.

Lu, Z. and Y. Dong (2011). "Fingering instability in forward smolder combustion". In: Combust. Theory Model. 15, pp. 795-815.

Lu, Z. et al. (2006). "Instabilities of reverse smolder waves". In: Combust. Theory Model. 10, pp. $515-534$.

Matalon, M. (2009). "Flame dynamics". In: Proc. Combust. Inst. 32, pp. 57-82.

Metzener, P. and M. Matalon (2006). "Diffusive-thermal instabilities of diffusion flames: onset of cells and oscillations". In: Combust. Theory Model. 10, pp. 701-725.

Miclavcic, M., A. B. Moore, and I. S. Wichman (2005). "Oscillations and island evolution in radiating diffusion flames". In: Combust. Theory Model. 9, pp. 403-416.

Ohki, Y. and S. Tsugé (1974). “On flame spreading over a polymer surface”. In: Combust. Sci. Technol. 9, pp. 1-12. 
Ohlemiller, T. J. (1985). "Modeling of smoldering combustion propagation". In: Prog. Energy Combust. Sci. 11, pp. 277-310.

Ohlemiller, T. J. and D. A. Lucca (1983). “An experimental comparison of forward and reyerse smolder propagation in permeable fuel beds". In: Combust. Flame 54, pp. 131-147.

Olson, S. L. (1991). "Mechanisms of microgravity flame spread over a thin solid fuel: oxygen and opposed flow effects”. In: Combust. Sci. Technol. 76, pp. 233-249.

Olson, S. L., P. V. Ferkul, and J. S. T'ien (1988). "Near-limit flame spread over a thin solid fuel in microgravity". In: Proc. Combust. Inst. 22, pp. 1213-1222.

Olson, S. L., H. R. Baum, and T. Kashiwagi (1998). "Finger-like smoldering over thin cellulosic sheets in microgravity”. In: Proc. Combust. Inst. 27, pp. 2525-2533.

Olson, S. L., F. J. Miller, and I. S. Wichman (2006). "Characterizing fingering flamelets using the logistic model". In: Combust. Theory Model. 10.2, pp. 323-347.

Olson, S. L. et al. (2009). "Flame spread over thin fuels in actual and simulated microgravity conditions". In: Combust. Flame 156, pp. 1214-1226.

Rein, G. (2009). "Smouldering combustion phenomena in science and technology". In: Intl. Rev. Chem. Eng. 1, pp. 3-18.

Schult, D. A. et al. (1996). "Forced forward smolder combustion". In: Combust. Flame 104, pp. $1-26$. 
Sohn, C. H., S. H. Chung, and J. S. Kim (1999). "Instability-induced extinction of diffusion flames established in the stagnant mixing layer". In: Combust. Flame 117, pp. 404-412.

Sohn, C. H. et al. (2000). "Nonlinear evolution of diffusion flame oscillations triggered by radiative heat loss". In: Combust. Flame 123, pp. 95-106.

T'ien, J. S. et al. (2001). "Mechanisms of flame spread and smolder wave propagation". In: Microgravity Combustion: Fire in Free Fall. Ed. by H. D. Ross. Academic Press, pp. 299-418.

Uchida, Y., K. Kuwana, and G. Kushida (2015). "Experimental validation of Lewis number and convection effects on the smoldering combustion of a thin solid in a narrow space". In: Combust. Flame 162, pp. 1957-1963.

Wichman, I. S. (1992). “Theory of opposed-flow flame spread”. In: Prog. Energy Combust. Sci. 18, pp. 553-593.

Williams, F. A. (1977). "Mechanisms of fire spread". In: Proc. Combust. Inst. 16, pp. 12811294.

Yuan, F. and Z. Lu (2013). "Structure and stability of non-adiabatic reverse smolder waves". In: Appl. Math. Mech.-Engl. Ed. 34, pp. 657-668.

Zhang, Y. et al. (1992). "Lewis number effects on flame spreading over thin solid fuels". In: Combust. Flame 90, pp. 71-83.

Zik, O. and E. Moses (1998). "Fingering instability in solid fuel combustion: the characteristic scales of the developed state". In: Proc. Combust. Inst. 27, pp. 2815-2820. 
Zik, O. and E. Moses (1999). "Fingering instability in combustion: an extended view". In: Phys. Rev. E 60, pp. 518-531.

Zik, O., Z. Olami, and E. Moses (1998). "Fingering instability in combustion". In: Phys. Rev. Lett. 81, pp. 3868-3871. 
Figure 1: Schematic of the experimental setup: (1) gas diffuser; (2) contraction section; (3) narrow channel; (4) fuel sample; (5) sample holder; (6) spring; (7) ignition wire. Top: top view; bottom: side view. Unit: mm.

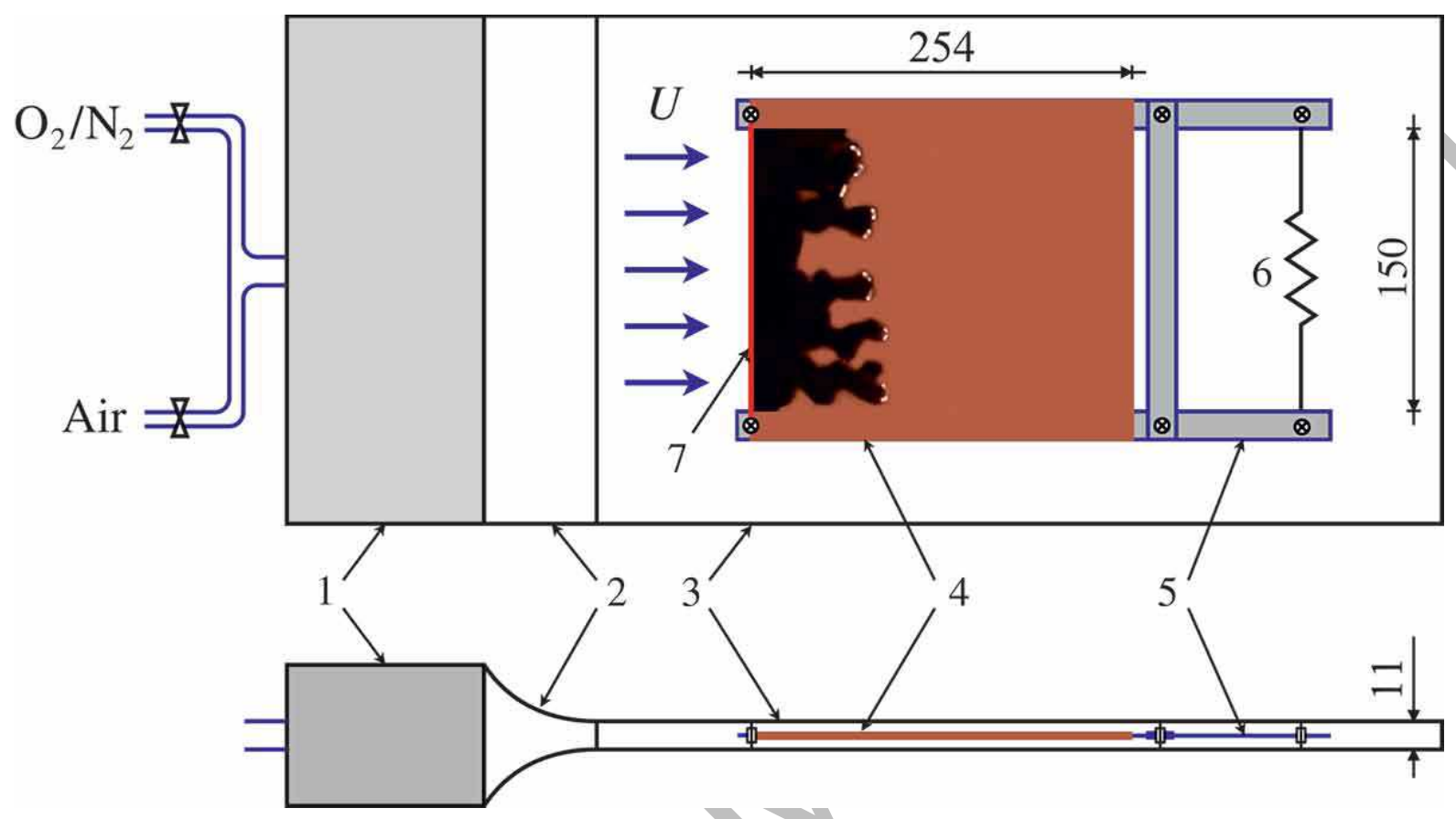


Figure 2: Snapshots showing the time evolution of concurrent flame spread in an $\mathrm{N}_{2} /$ Air atmosphere. Ambient oxygen concentration $C_{\mathrm{Of}}=15 \%$; gas flow velocity $U=6 \mathrm{~cm} / \mathrm{s}$. Gas blows from bottom to top. Times from ignition are (bottom to top) 20.5, 54.5, 63.1 and 92.1s. The fuel sample was illuminated by a red LED light during the test.

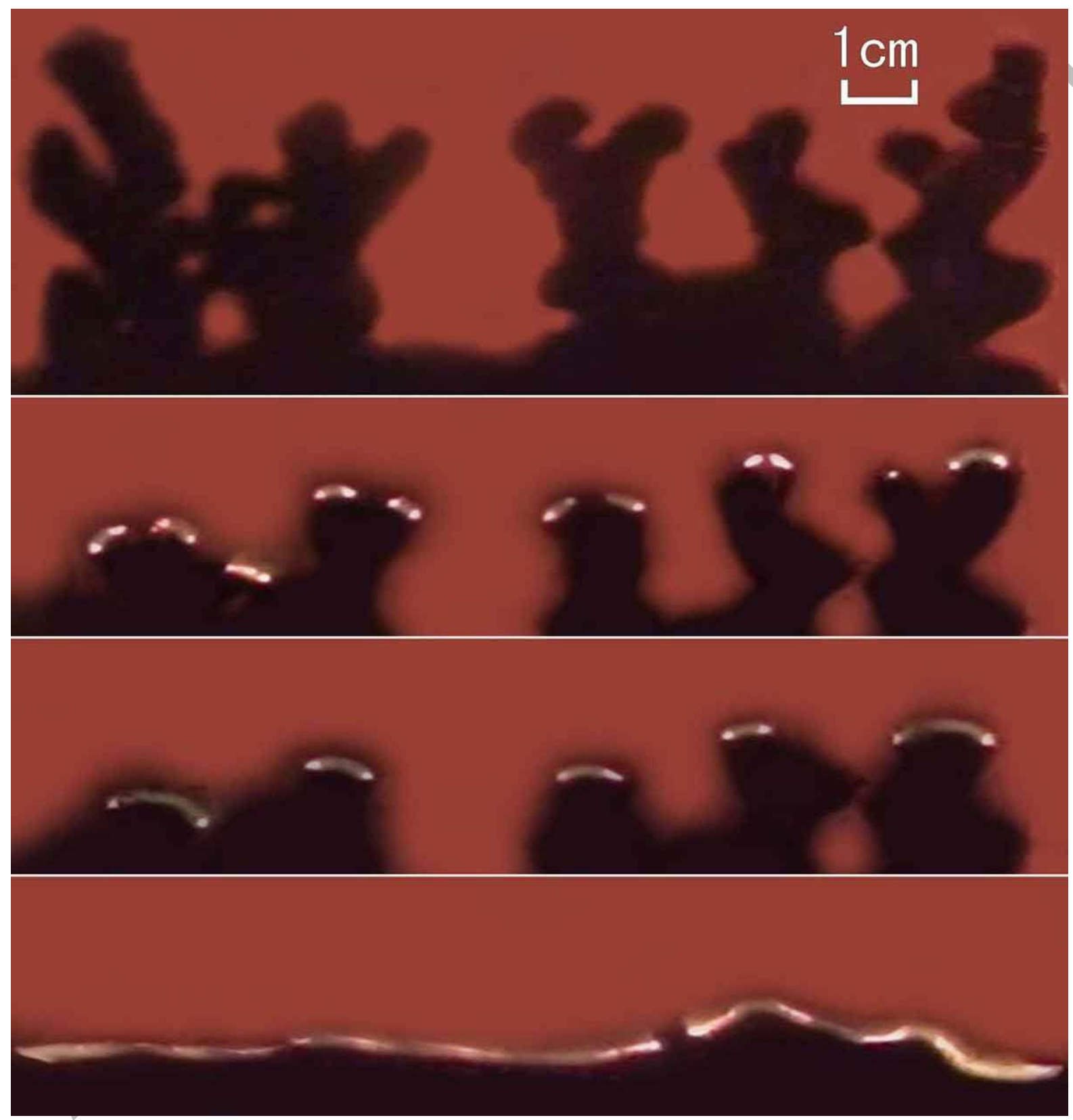


Figure 3: Snapshots showing the time evolution of concurrent flame spread in an $\mathrm{N}_{2} /$ Air atmosphere. Ambient oxygen concentration $C_{\mathrm{Of}}=15 \%$; gas flow velocity $U=7 \mathrm{~cm} / \mathrm{s}$. Gas blows from bottom to top. Times from ignition are (a): 10.0s, (b): $68.4 \mathrm{~s}$, (c): $75.2 \mathrm{~s}$, and (d): $120.2 \mathrm{~s}$. The fuel sample was illuminated by a red LED light during the test.

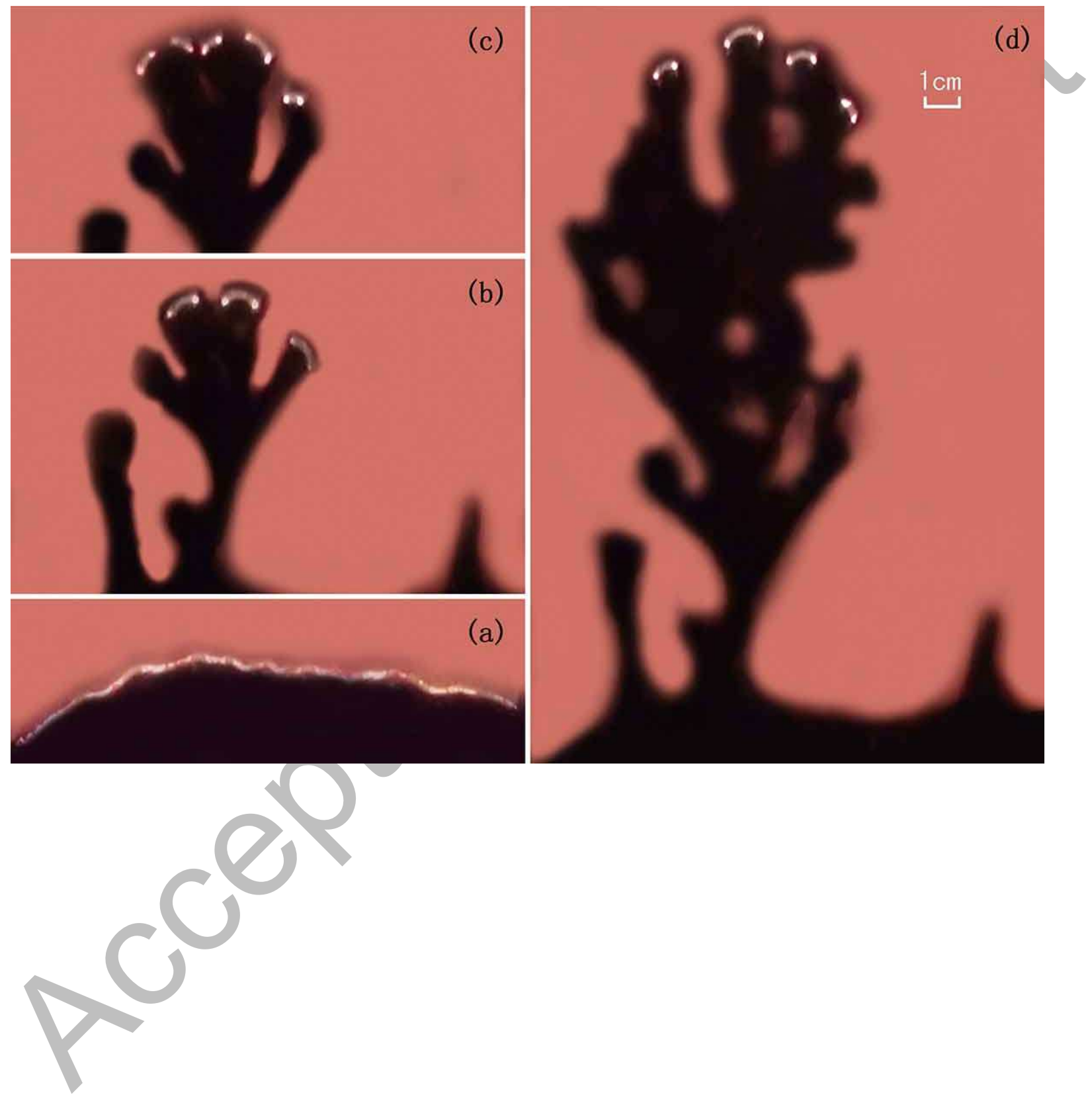


Figure 4: Bifurcation diagram showing the evolution of flame spread for the case shown in Fig. 3. Solid lines correspond to branches that eventually burn through the fuel sample; dashed lines correspond to branches that eventually extinguish; filled circle symbols mark the points where flamelets split. Arrows indicate the direction of gas flow.

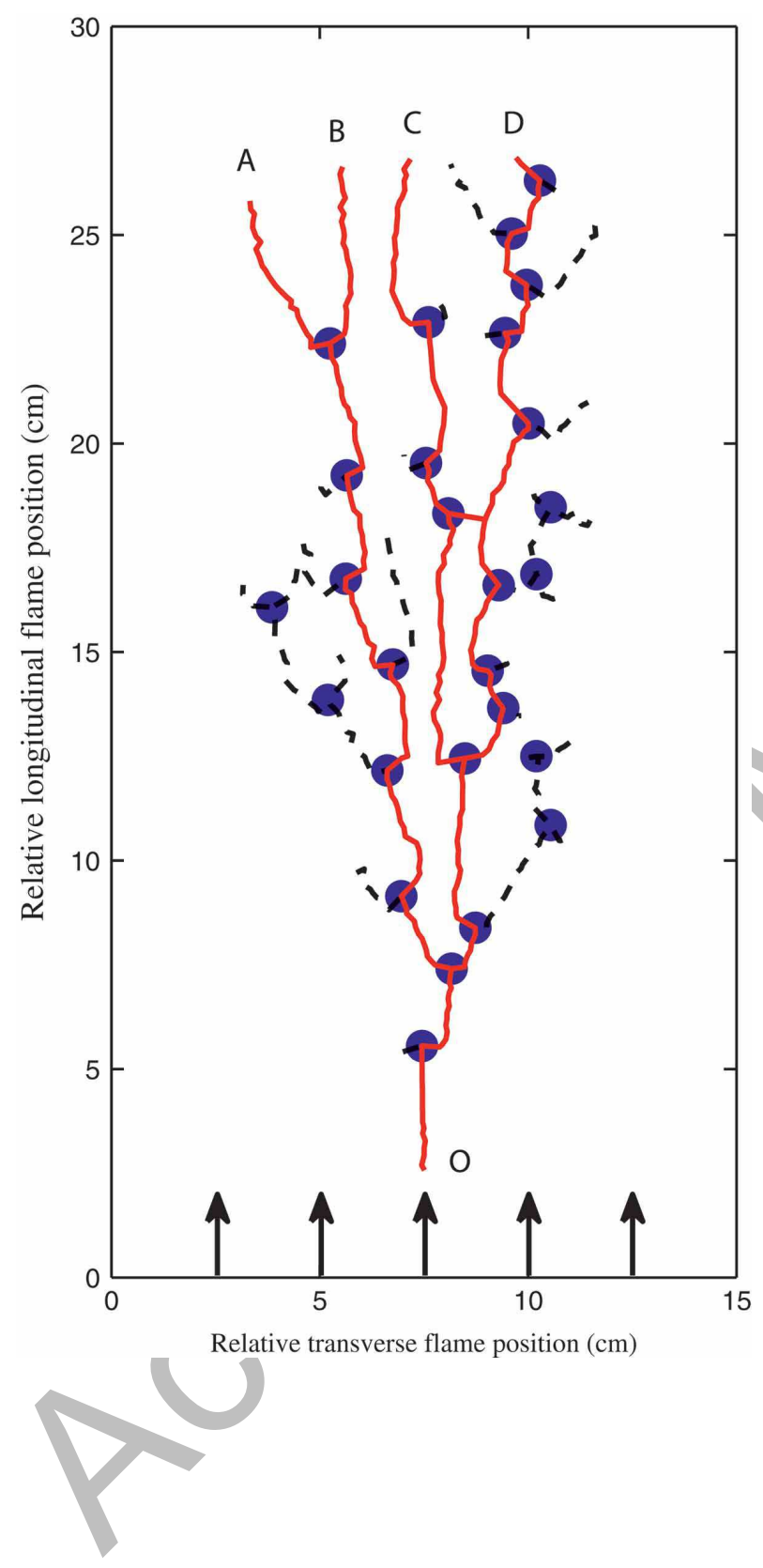


Figure 5: Time evolution of longitudinal flame position and flamelet width along branch $O C$ as delineated in Fig. 4. Dashed line corresponds to linear fitting of the flame position data, yielding a flame spread rate $V_{\mathrm{f}} \approx 0.18 \mathrm{~cm} / \mathrm{s}$. Filled symbols mark the bifurcation points.

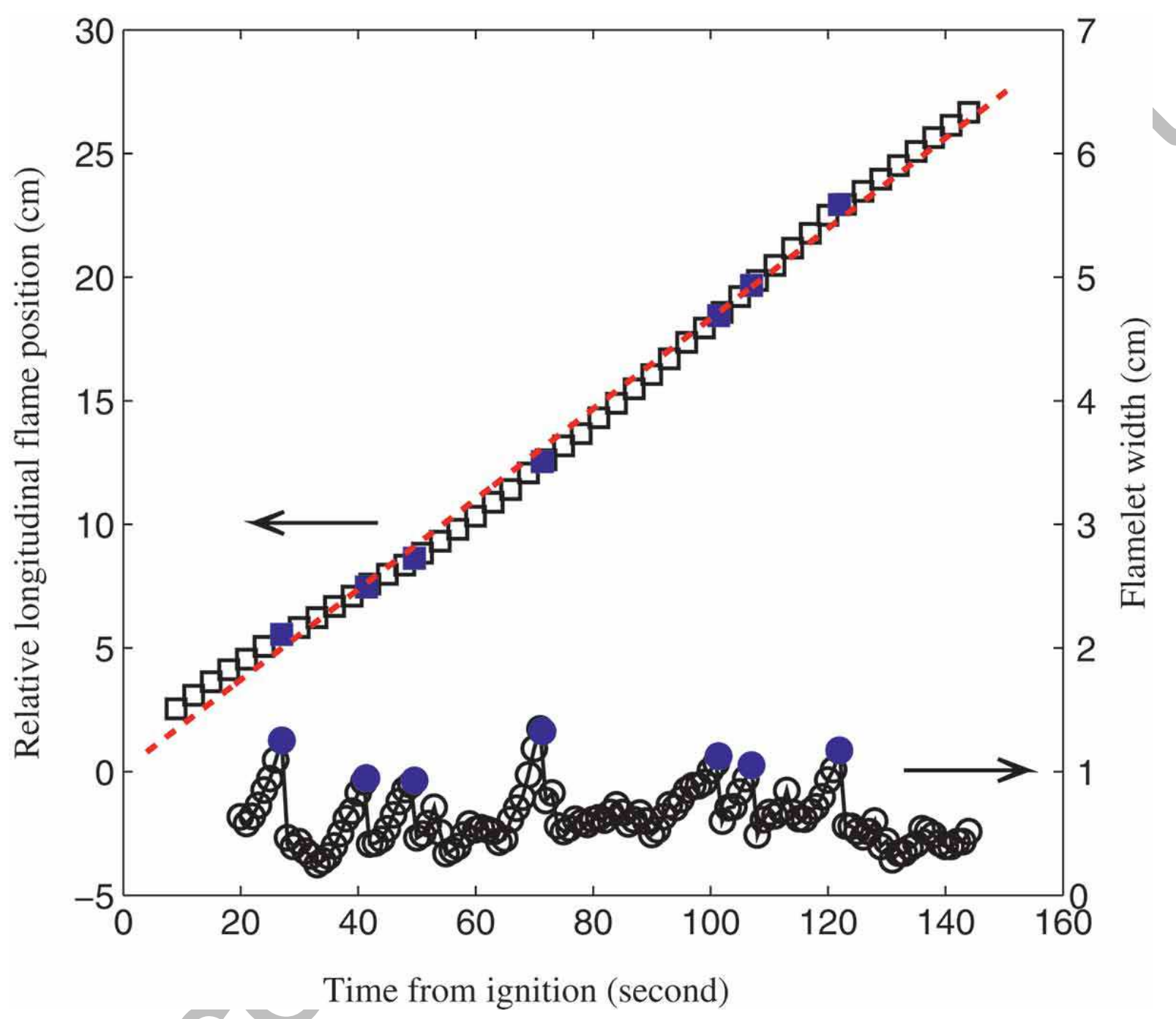


Figure 6: Snapshots showing the time evolution of concurrent flame spread in an $\mathrm{N}_{2} /$ Air atmosphere. Ambient oxygen concentration $C_{\mathrm{Of}}=15 \%$; gas flow velocity $U=9 \mathrm{~cm} / \mathrm{s}$. Gas blows from bottom to top. Times from ignition are (bottom to top) $9.9 \mathrm{~s}$ and $59.4 \mathrm{~s}$, respectively. The fuel sample was illuminated by a red LED light during the test.

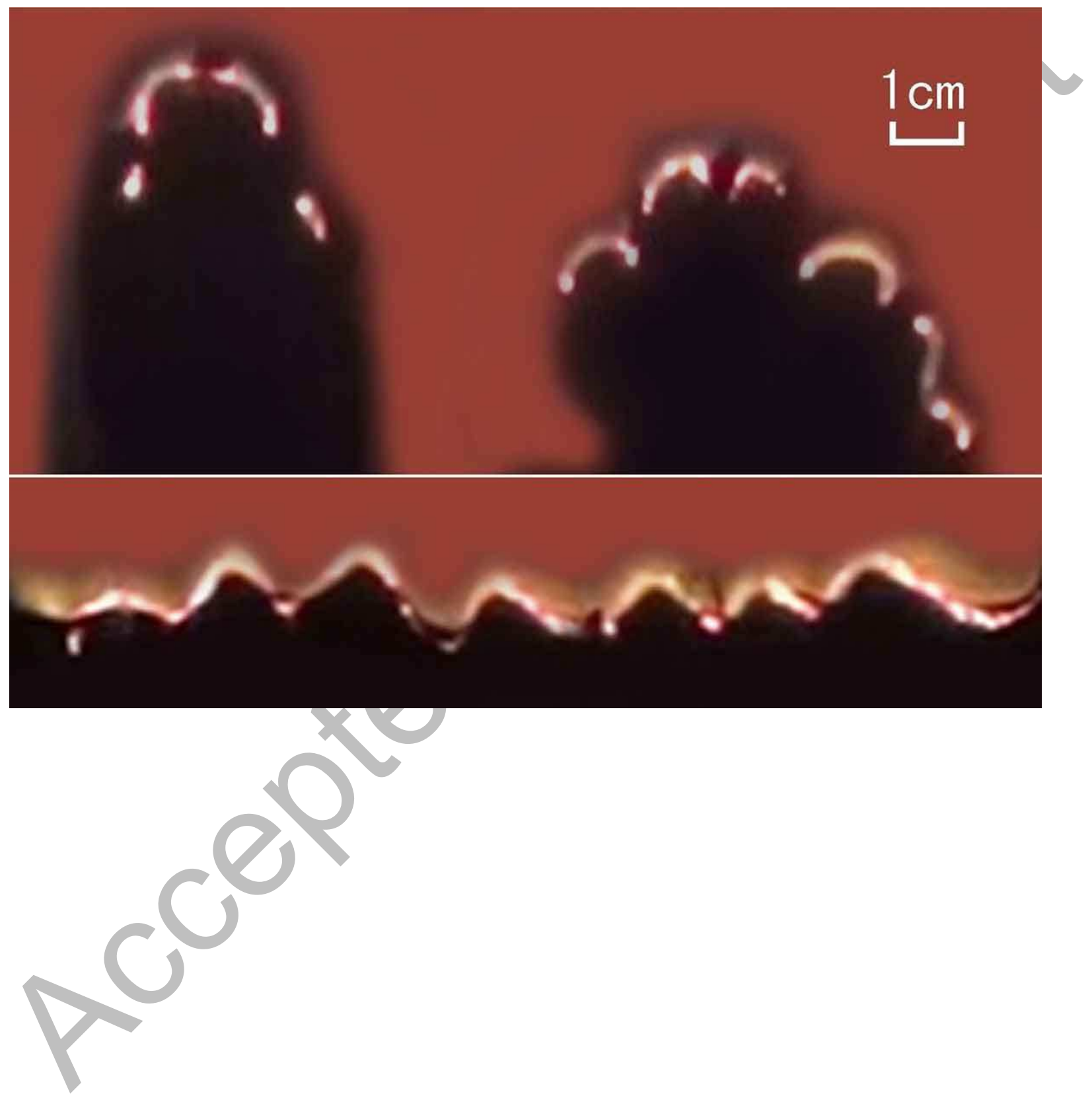


Figure 7: Snapshots showing the time evolution of concurrent flame spread in an $\mathrm{N}_{2} /$ Air atmosphere. Ambient oxygen concentration $C_{\mathrm{Of}}=15 \%$; gas flow velocity $U=17 \mathrm{~cm} / \mathrm{s}$. Gas blows from bottom to top. Times from ignition are (bottom to top) $8.9 \mathrm{~s}$ and $20.4 \mathrm{~s}$, respectively. The fuel sample was illuminated by a red LED light during the test.

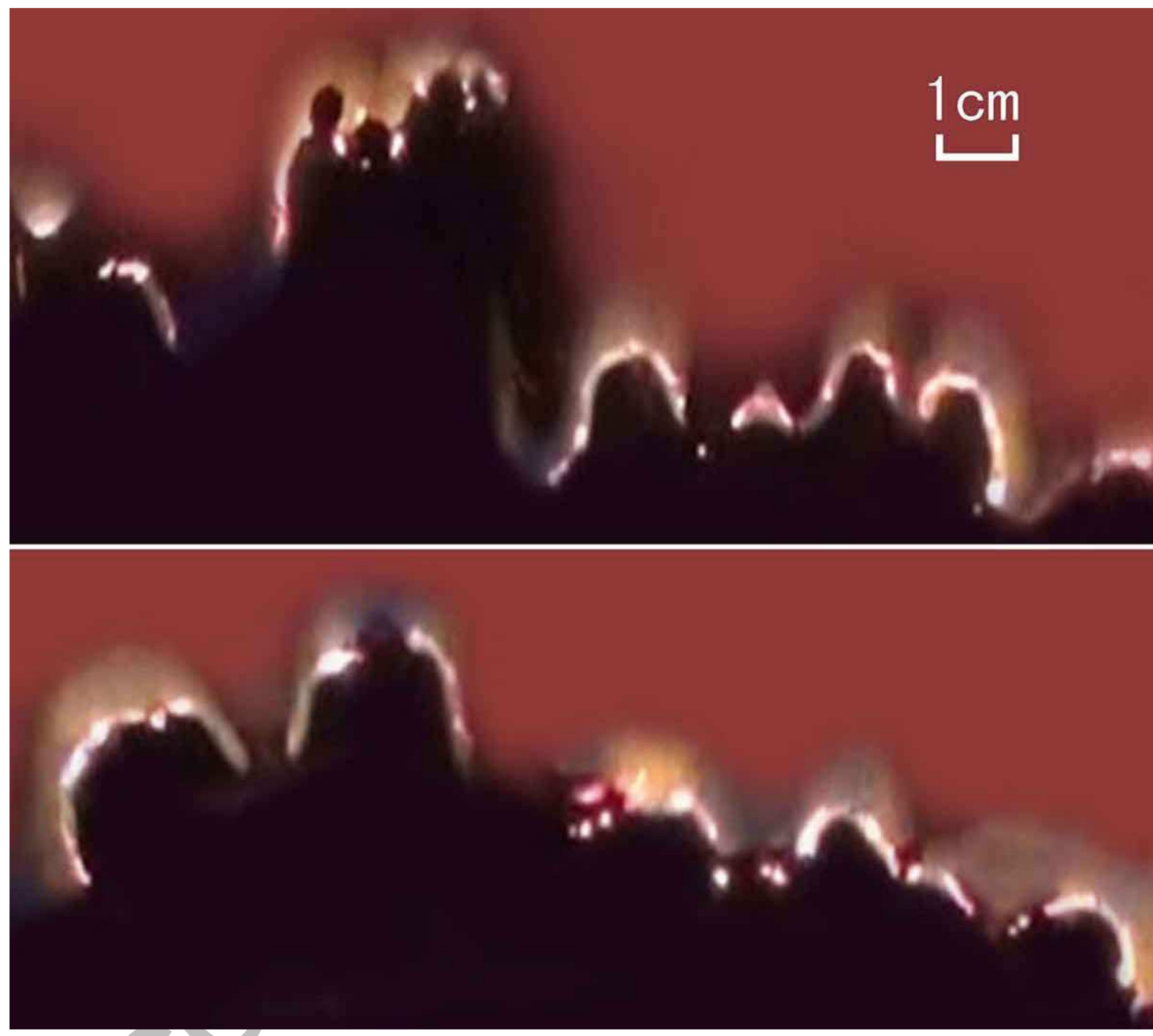


Figure 8: Flame spread rate and critical flamelet width as a function of imposed gas flow velocity at $15 \%$ oxygen. Circle symbols represent flame spread rates corresponding to the fastest spreading flamelets; square symbols and error bars correspond to mean and standard deviation of the critical flamelet width data.

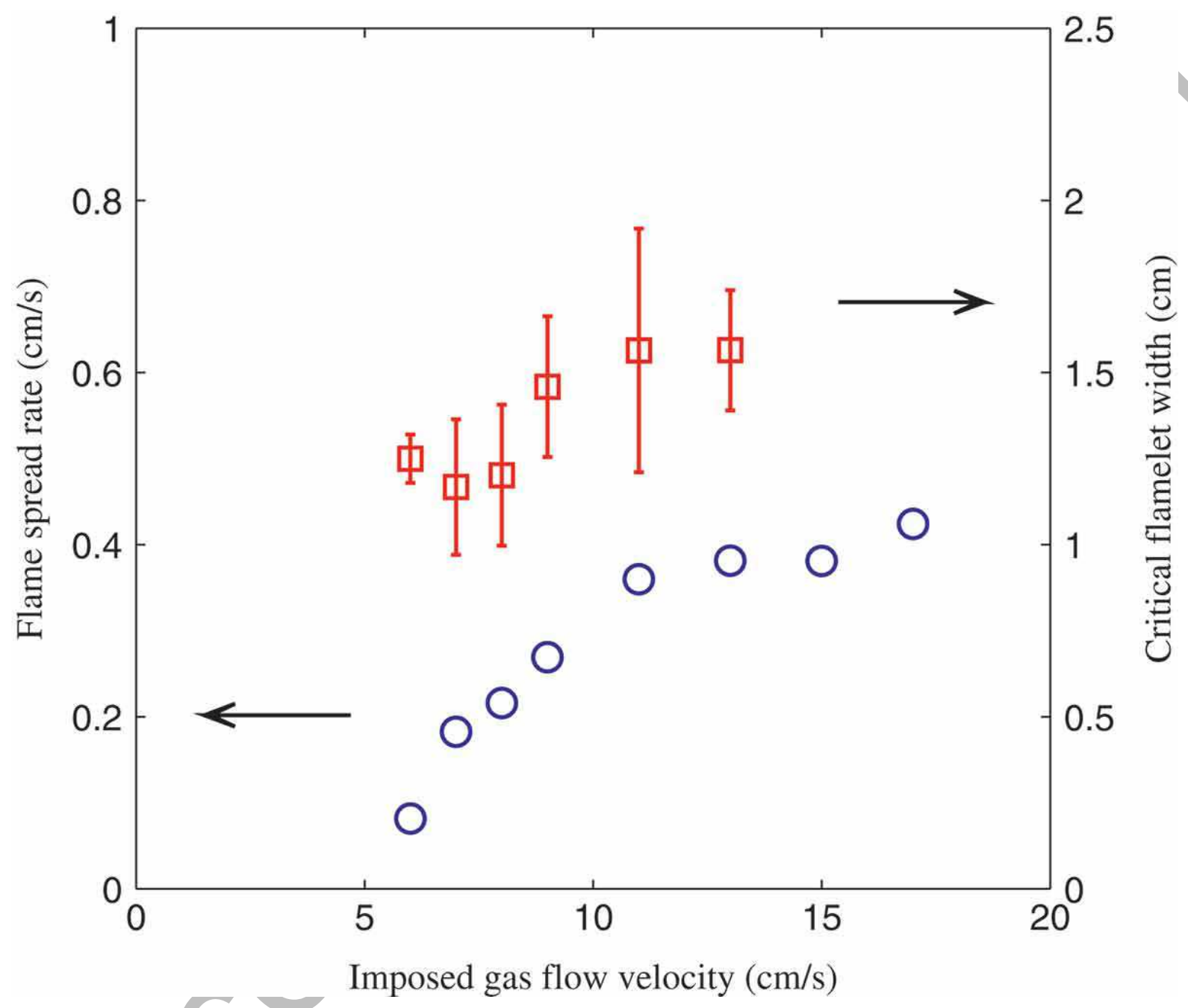


Figure 9: Snapshots showing the time evolution of concurrent flame spread in air with gas flow velocity $U=3 \mathrm{~cm} / \mathrm{s}$. Gas blows from bottom to top. Times from ignition are (bottom to top) $24.6,33.6,35.6,41.6,45.1,48.6,63.1$ and $64.6 \mathrm{~s}$, respectively.

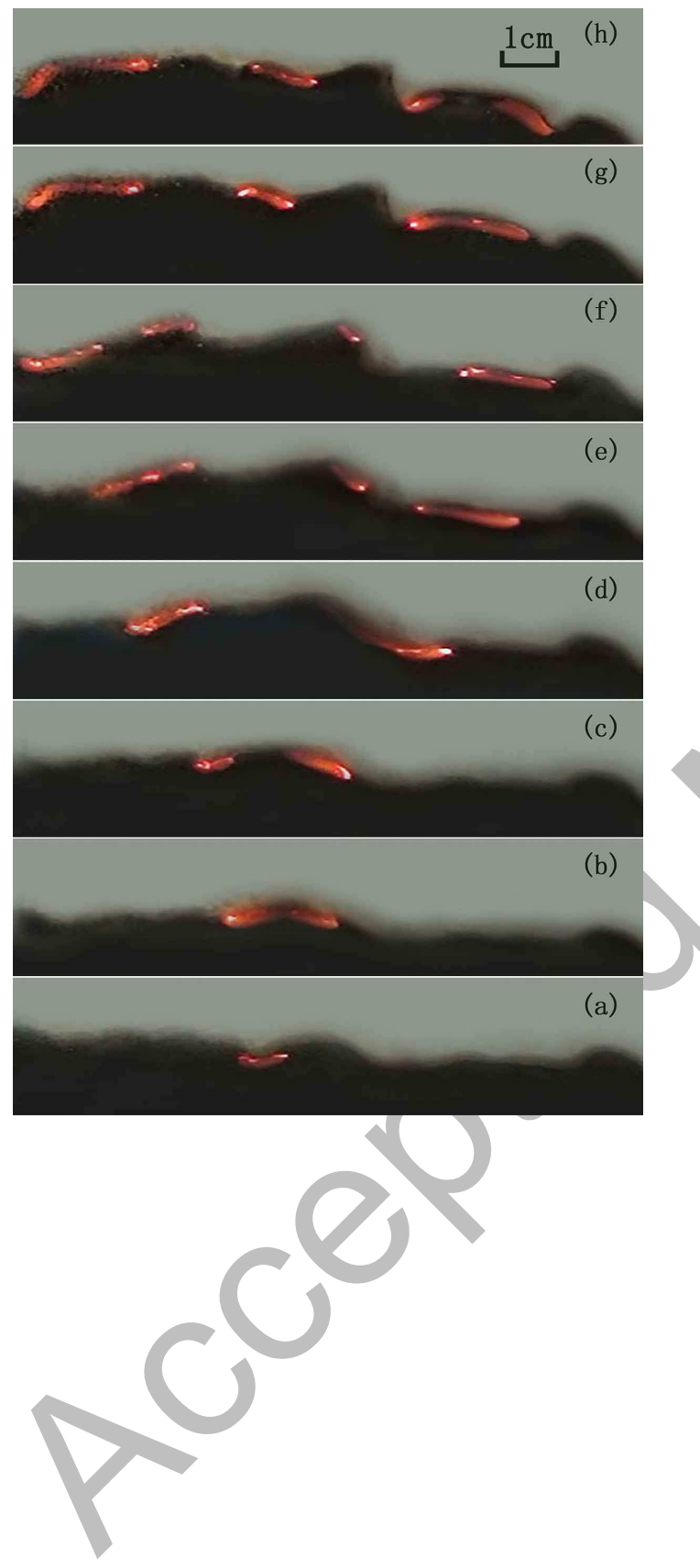


Figure 10: Time evolution of transverse flame edge positions for the case shown in Fig. 9. (a) through (h) mark the instants that correspond to the flame images shown in Fig. 9. Filled circle symbols mark the points where flamelets split. Vertical solid and dotted lines correspond to transverse position $y=5.49 \mathrm{~cm}$.

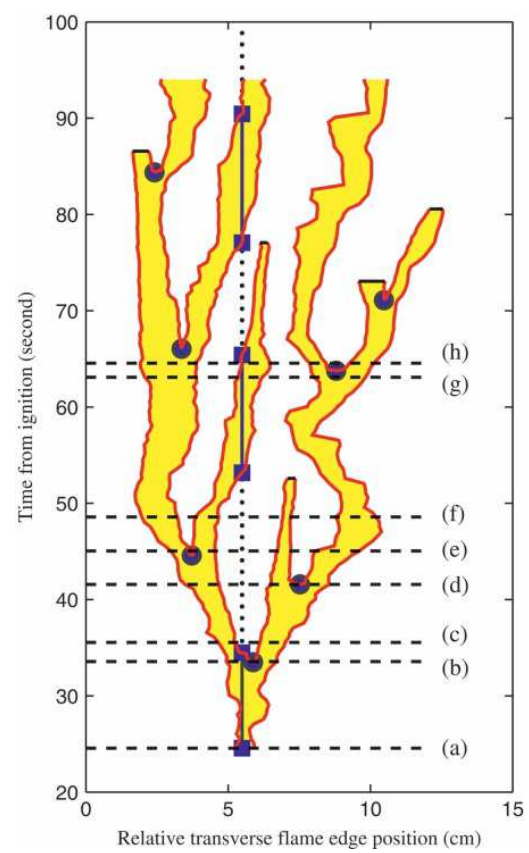


Figure 11: Time evolution of longitudinal flame position for the case shown in Fig. 9. Transverse reference position is fixed at $y=5.49 \mathrm{~cm}$ (cf. Fig. 10). Solid and dotted lines correspond, respectively, to time portions when flamelets are present and absent. Dashed line represents linear fit of the entire flame position data, whose slope defines the nominal flame spread rate. The actual flame spread rate is determined by linear fit of the solid line segments.

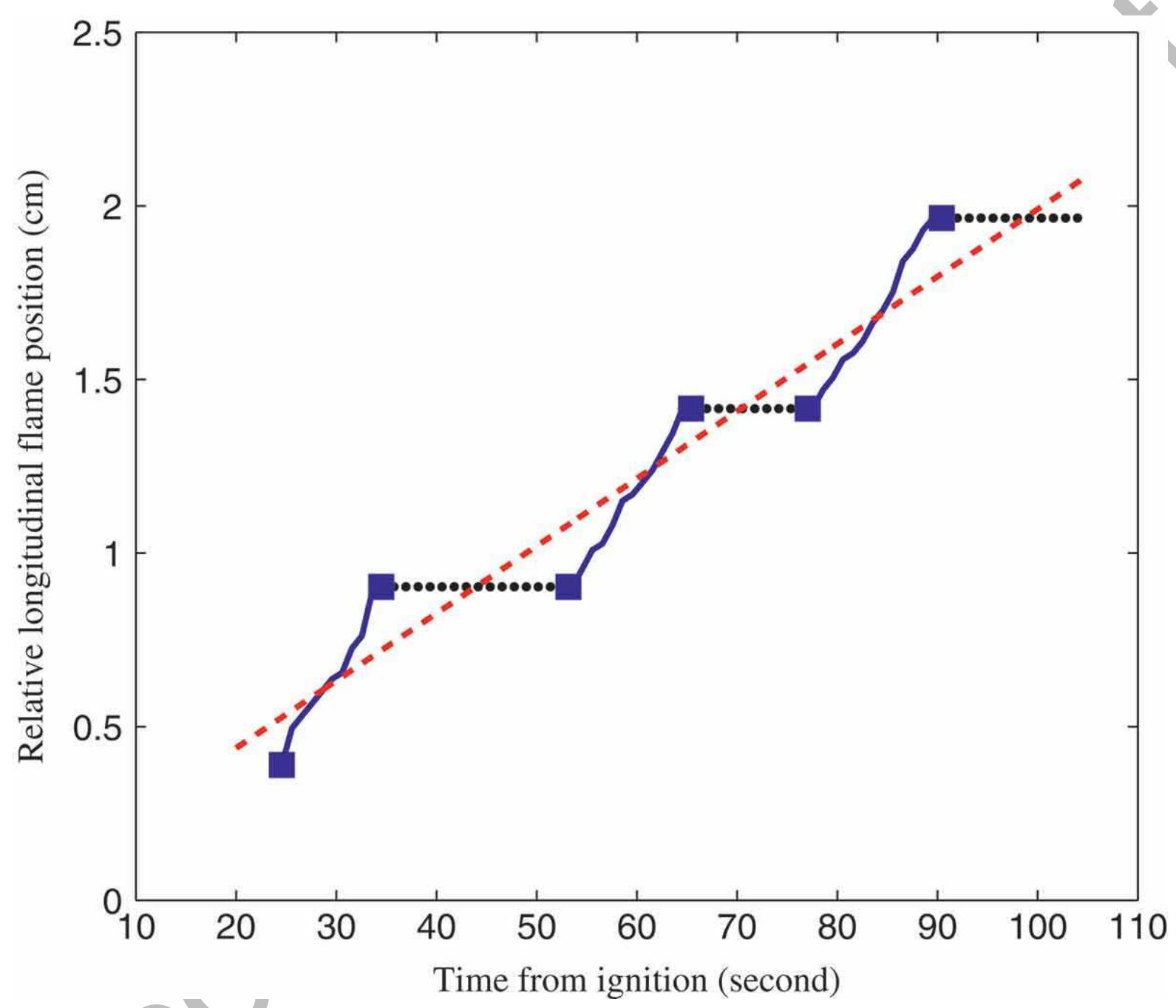


Figure 12: Snapshots showing the time evolution of concurrent flame spread in air with gas flow velocity $U=5 \mathrm{~cm} / \mathrm{s}$. Gas blows from bottom to top. Times from ignition are (bottom to top) $10.1,81.1$ and 99.1 s, respectively.

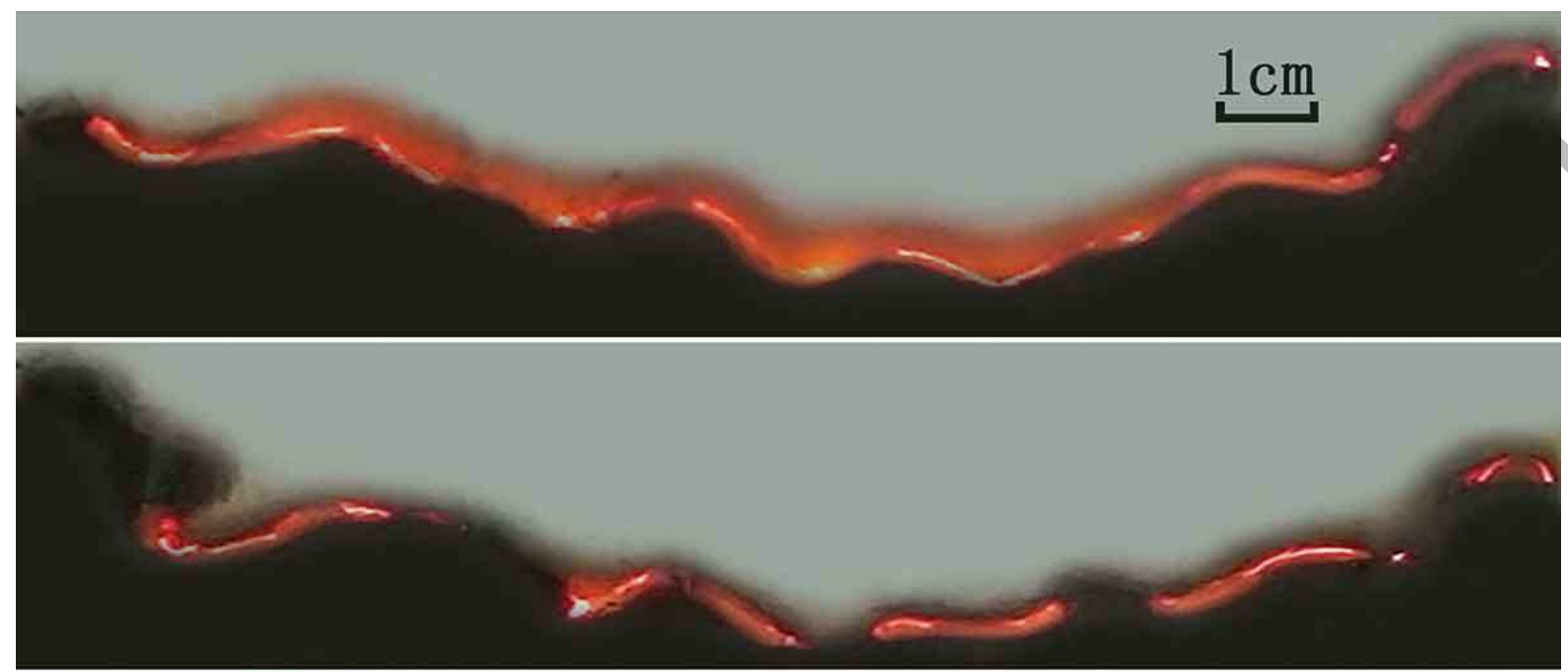


Figure 13: Flammability map and stability diagram of concurrent flame spread in an $\mathrm{O}_{2} / \mathrm{N}_{2} / \mathrm{Air}$ atmosphere. The $\nabla$ symbols mark the cases that correspond to fingering or cellular instability (FCI), $\Delta$ corresponds to travelling wave instability (TWI), $\diamond$ corresponds to transitional state between FCI and TWI, and $\square$ corresponds to stable flame spread. The black-filled symbols mark the cases that are non-flammable, and the is symbols correspond to incomplete flame spread. The solid line that is obtained by curve fitting corresponds to the flammability boundary, and the fitted dashed line approximates the marginal stability boundary that separates the stable and unstable flame spread regimes. The dotted line segment that corresponds to a critical oxygen concentration, $C_{\mathrm{Oc}} \approx 19 \%$, represents the boundary that separates FCI and TWI.

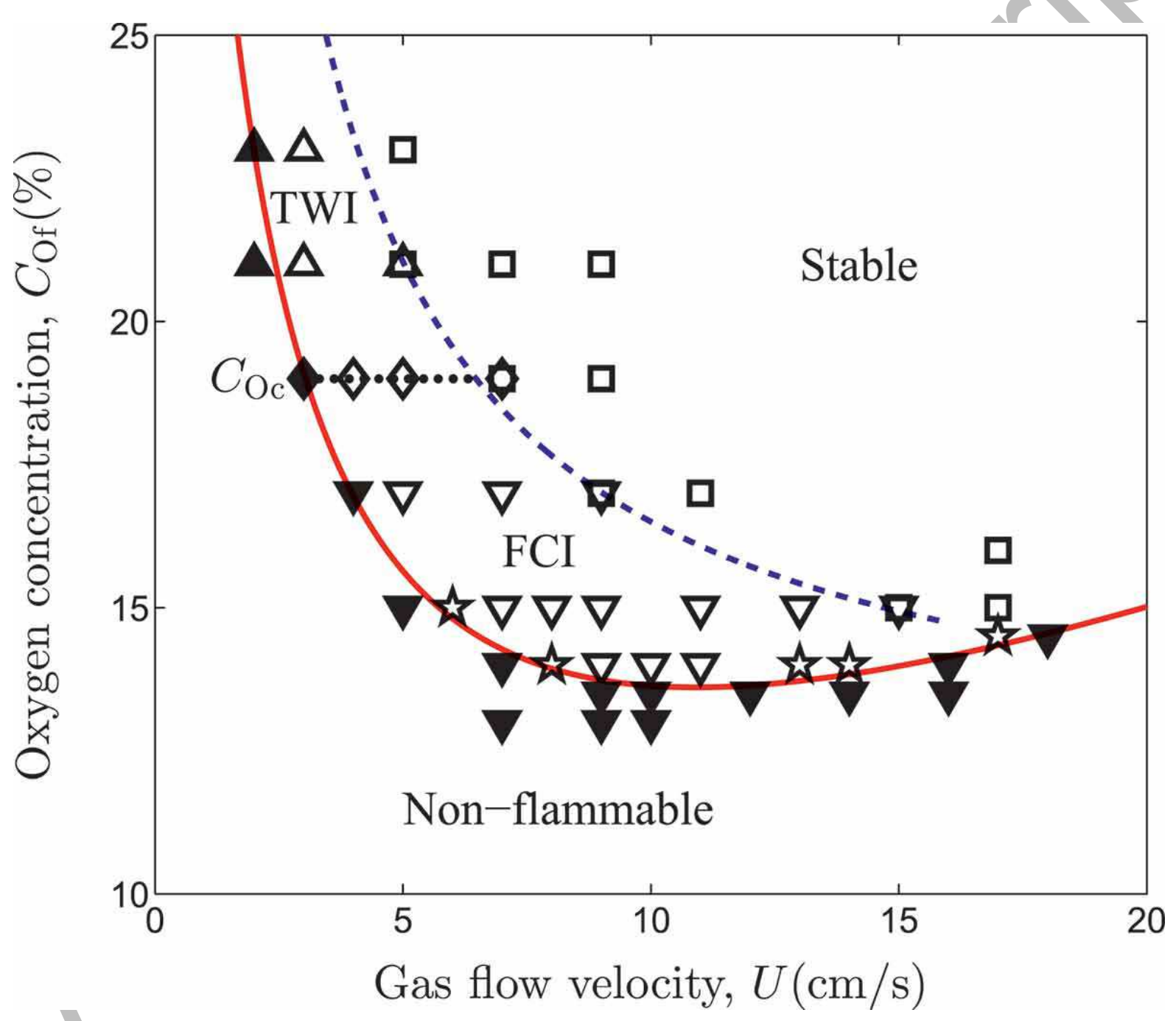


Figure 14: Snapshots showing the time evolution of concurrent flame spread in an $\mathrm{N}_{2} /$ Air atmosphere. Ambient oxygen concentration $C_{\mathrm{Of}}=19 \%$; gas flow velocity $U=5 \mathrm{~cm} / \mathrm{s}$. Gas blows from bottom to top. Times from ignition are (bottom to top) 8.1, 28.6, 31.2, 41.8 and 43.7s, respectively. Circles mark the flamelets that crawl back along the finger walls.

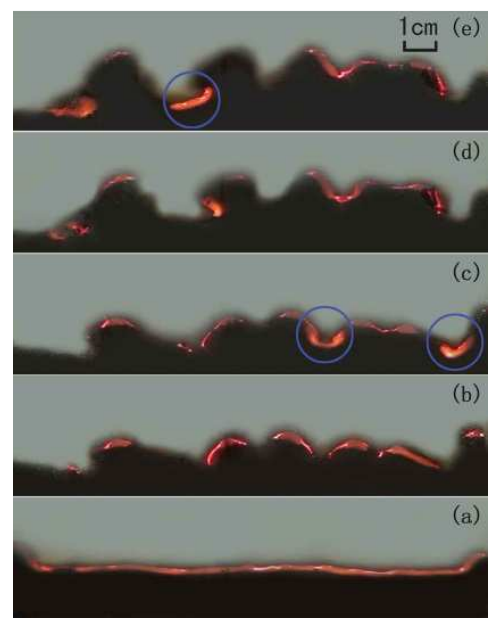

